\title{
Electron acoustic solitons in the Earth's magnetotail
}

\author{
S. G. Tagare ${ }^{1}$, S. V. Singh ${ }^{2,3}$, R. V. Reddy ${ }^{2}$, and G. S. Lakhina ${ }^{2,4}$ \\ ${ }^{1}$ Applied Mathematics, Shadan Institue of P. G. Studies, Khairatabad, Hyderabad 5000004, India \\ ${ }^{2}$ Indian Institute of Geomagnetism, Plot No. 5, Sector-18, New Panvel (W), Navi Mumbai - 410218, India \\ ${ }^{3}$ Radio Science Center for Space \& Atmosphere, Kyoto University, Kyoto 611-0011, Japan \\ ${ }^{4}$ Solar-Terrestrial Environment Laboratory, Nagoya University, Toyokawa, Japan
}

Received: 8 August 2003 - Revised: 5 December 2003 - Accepted: 17 December 2003 - Published: 14 April 2004

Part of Special Issue "International Workshops on Nonlinear Waves and Chaos in Space Plasmas"

\begin{abstract}
Small amplitude electron - acoustic solitons are studied in a magnetized plasma consisting of two types of electrons, namely cold electron beam and background plasma electrons and two temperature ion plasma. The analysis predicts rarefactive solitons. The model may provide a possible explanation for the perpendicular polarization of the low-frequency component of the broadband electrostatic noise observed in the Earth's magnetotail.
\end{abstract}

\section{Introduction}

Intense broadband electrostatic noise (BEN) are commonly observed in the plasma sheet boundary layer (PSBL) of the Earth's magnetosphere (Scarf et al., 1974; Gurnett et al., 1976). The frequency of these BENs range from $\sim 10 \mathrm{~Hz}$ up to the local electron plasma frequency $\sim 10 \mathrm{kHz}$ and electric field intensities from $\sim$ few $\mathrm{mV} / \mathrm{m}$ to $50 \mathrm{mV} / \mathrm{m}$ and higher. These BENs are observed in conjunction with the ion beams. However, there are observations which show the presence of electron beams in the PSBL and their correlation with the BEN (Parks et al., 1984; Onsager et al., 1993). Matsumoto et al. (1994) have shown that broadband electrostatic noise emissions in the plasma sheet boundary layer are not continuous noise but consists of electrostatic impulsive solitary waves. The data from Polar satellite shows that at the high altitude cusp, polar cap and plasma sheet boundary layer, the solitary waves are positive potential structures (electron holes) and propagate with velocities of a few thousand $\mathrm{km} / \mathrm{s}$ (Cattell et al., 1999). The scale sizes of these structures are of the order of tens of Debye lengths. It is found that the solitary waves in the plasma sheet boundary layer are an electron mode waves, either an electron hole or an electron-acoustic solitary wave.

Several studies on nonlinear electron-acoustic waves have been done in the past (Buti, 1980; Buti et al., 1980; Yu and

Correspondence to: S. V. Singh

(satyavir@iigs.iigm.res.in)
Shukla, 1983; Guha and Dwivedi, 1984; Mace et al., 1991). Buti (1980) studied the propagation of electron-acoustic solitary waves and holes in magnetized plasma with electron and two Boltzmann distributed ion species. Guha and Dwivedi (1984) examined the linear and nonlinear propagation of electron-acoustic waves with two electrons and two ions in an unmagnetized plasma.

The observations (Parks et al., 1984; Onsager et al., 1993) in the plasma sheet boundary layer have shown that there exist two types of electrons, namely background plasma electrons and cold electron beams having energies of the order of few $\mathrm{eV}$ to few hundreds of $\mathrm{eV}$ and two different species of ions, namely background cold ions and warm ions/ion beams with energies from few $\mathrm{keV}$ to tens of $\mathrm{keV}$.

Motivated from these observations, we examine the generation of small amplitude electron-acoustic solitons in a magnetized plasma with four components namely, cold electron beam and background plasma electrons and two temperature ion plasma. We treat both the electron species as cool in comparison to more energetic ions. The effect of various parameters such as soliton velocity, electron beam drift speed, is studied on the soliton amplitude. In the next section, formulation of the problem is discussed and results are concluded in the last section.

\section{Formulation}

We consider a homogeneous plasma with two types of electrons, namely, cold electron beam and background plasma electrons and two ion species in an external magnetic field $\boldsymbol{B}_{\mathbf{0}}$ which is assumed to be in the $x-z$ plane. Here, the ions are taken as unmagnetized and are assumed to have Boltzmann distribution. These assumptions are valid when the gyroperiods and Larmor radii of both the ion species are much larger than the wave period and the wavelength (Buti, 1980). The electron inertia plays an important role in the electronacoustic wave generation, therefore, electron dynamics is governed by the fluid equations. They are treated magnetized 
as the electron-acoustic frequencies are smaller than the electron cyclotron frequencies. Thermal effects for both the electron species are neglected. The electron-acoustic waves are considered to be propagating along $x$-direction.

The basic set of equations for the electrons and ions are given by

$\frac{\partial n_{s}}{\partial t}+\frac{\partial\left(n_{s} u_{s x}\right)}{\partial x}=0$

$\frac{\partial u_{s x}}{\partial t}+u_{s x} \frac{\partial u_{s x}}{\partial x}=\frac{\partial \phi}{\partial x}+u_{s y} \sin \theta$

$\frac{\partial u_{s y}}{\partial t}+u_{s x} \frac{\partial u_{s y}}{\partial x}=u_{s z} \cos \theta-u_{s x} \sin \theta$

$\frac{\partial u_{s z}}{\partial t}+u_{s x} \frac{\partial u_{s z}}{\partial x}=-u_{s y} \cos \theta$

$n_{l}=\mu \exp \left(\frac{-\phi}{\mu+\nu \beta}\right)$

$n_{h}=v \exp \left(\frac{-\beta \phi}{\mu+v \beta}\right)$

and the Poisson's equation is given by

$\kappa \frac{\partial^{2} \phi}{\partial z^{2}}=n_{e}+n_{b}-n_{l}-n_{h}$

Here

$\kappa=\frac{T_{e f}}{4 \pi n_{0} \rho_{e}^{2} e^{2}}, \quad \rho_{e}=\frac{C_{S}}{\Omega_{e}}, \quad \beta=\frac{T_{l}}{T_{h}}$,

$T_{e f}=\frac{T_{l} T_{h}}{\mu T_{h}+v T_{l}}, \quad C_{S}=\left(T_{e f} / m_{e}\right)^{1 / 2}$,

and $\mu+v=1$,

and $\theta$ is the angle between the direction of propagation of the wave and magnetic field $\boldsymbol{B}_{\mathbf{0}}$. The subscript $s=e, b$ refers to background plasma electrons, and cold beam electrons respectively, and $i=l, h$ refers to cold - temperature $\left(T_{l}\right)$ and hot - temperature $\left(T_{h}\right)$ ions respectively and $\phi$ is the electrostatic potential. The physical parameters $x, t,\left(n_{s}, n_{i}\right),\left(u_{s x}, u_{s y}, u_{s z}\right)$ and $\phi$ are normalized to effective electron cyclotron radius $\rho_{e}$, inverse of electron cyclotron frequency $\Omega_{e}^{-1}$, unperturbed plasma density $n_{0}$, effective electron acoustic velocity $C_{S}$ and effective potential $T_{e f} / e$ respectively. $\mu$ and $v$ are normalized equilibrium densities of low and high temperature ions respectively. We assume the boundary conditions, $n_{e} \rightarrow 1-\alpha, n_{b} \rightarrow \alpha$, $u_{e} \rightarrow 0, u_{b x} \rightarrow u_{0} \cos \theta, u_{b y} \rightarrow 0, u_{b z} \rightarrow u_{0} \sin \theta, \phi \rightarrow 0, n_{l} \rightarrow \mu$, $n_{h} \rightarrow v$ as $|x| \rightarrow \infty$. Here $\alpha$ is the normalized density of beam electrons and $u_{0}$ denotes the normalized electron - beam velocity in the equilibrium state. In the next section, we study the propagation of rarefactive electron-acoustic solitons.

\section{Rarefactive solitons}

To solve the Eqs. (1)-(7) for soliton solutions, we shall use the reductive perturbation method (Washimi and Taniuti, 1966; Reddy and Lakhina, 1991). We introduce the stretched variables

$\xi=\epsilon^{1 / 2}(x-V t), \tau=\epsilon^{3 / 2} t$

where the small parameter $\epsilon$ is to be regarded as a measure of the strength of the nonlinearity and $V$ is the phase velocity. We expand the dependent variables of Eqs. (1)-(6) around the equilibrium state as follows:

$n_{e}=1-\alpha+\epsilon n_{e 1}+\epsilon^{2} n_{e 2}+\cdots$,
$n_{b}=\alpha+\epsilon n_{b 1}+\epsilon^{2} n_{b 2}+\cdots$,
$u_{e x}=\epsilon u_{e x 1}+\epsilon^{2} u_{e x 2}+\cdots$,
$u_{e y}=\epsilon^{3 / 2} u_{e y 1}+\epsilon^{5 / 2} u_{e y 2}+\cdots$,
$u_{e z}=\epsilon u_{e z 1}+\epsilon^{2} u_{e z 2}+\cdots$
$u_{b x}=u_{0} \cos \theta+\epsilon u_{b x 1}+\epsilon^{2} u_{b x 2}+\cdots$,
$u_{b y}=\epsilon^{3 / 2} u_{b y 1}+\epsilon^{5 / 2} u_{b y 2}+\cdots$
$u_{b z}=u_{0} \sin \theta+\epsilon u_{b z 1}+\epsilon^{2} u_{b z 2}+\cdots$,
and $\phi=\epsilon \phi_{1}+\epsilon^{2} \phi_{2}+\cdots$

Substituting Eqs. (8) and (9) in the system of Eqs. (1)-(7) and equating coefficients of same powers of $\epsilon$, to the lowest power of $\epsilon$ we get the following relation for the phase velocity, $V$,

$\frac{1-\alpha}{V^{2}}+\frac{\alpha}{\left(V-u_{0} \cos \theta\right)^{2}}=\sec ^{2} \theta$

For $u_{0}=0$ Eq. (10) gives a linear dispersion relation for electron-acoustic waves as $\omega=k C_{S} \cos \theta$ which becomes identical to that derived by Buti (1980) in the limit of cold electrons and $k \rho_{e}<<1$. To the next order of $\epsilon$, and by eliminating the second order quantities we get the usual $\mathrm{KdV}$ equation

$\frac{\partial \phi_{1}}{\partial \tau}+A \phi_{1} \frac{\partial \phi_{1}}{\partial \xi}+\frac{B}{2} \frac{\partial^{3} \phi_{1}}{\partial \xi^{3}}=0$

where

$A=\frac{1}{2 \cos ^{2} \theta\left(\frac{1-\alpha}{V^{3}}+\frac{\alpha}{\left(V-u_{0} \cos \theta\right)^{3}}\right)}$
$\cdot\left[\frac{\mu+v \beta^{2}}{(\mu+v \beta)^{2}}-3 \cos ^{4} \theta\left(\frac{1-\alpha}{V^{4}}+\frac{\alpha}{\left(V-u_{0} \cos \theta\right)^{4}}\right)\right]$

and

$B=\frac{\kappa+\sin ^{2} \theta}{\left(\frac{1-\alpha}{V^{3}}+\frac{\alpha}{\left(V-u_{0} \cos \theta\right)^{3}}\right)}$

Substituting $\chi=\xi-M \tau$ in Eq. (11); $M$ is the arbitrary parameter similar to Mach number which allows the possibility of solitons moving with different velocity than the phase velocity of the wave, and integrating Eq. (11) twice 


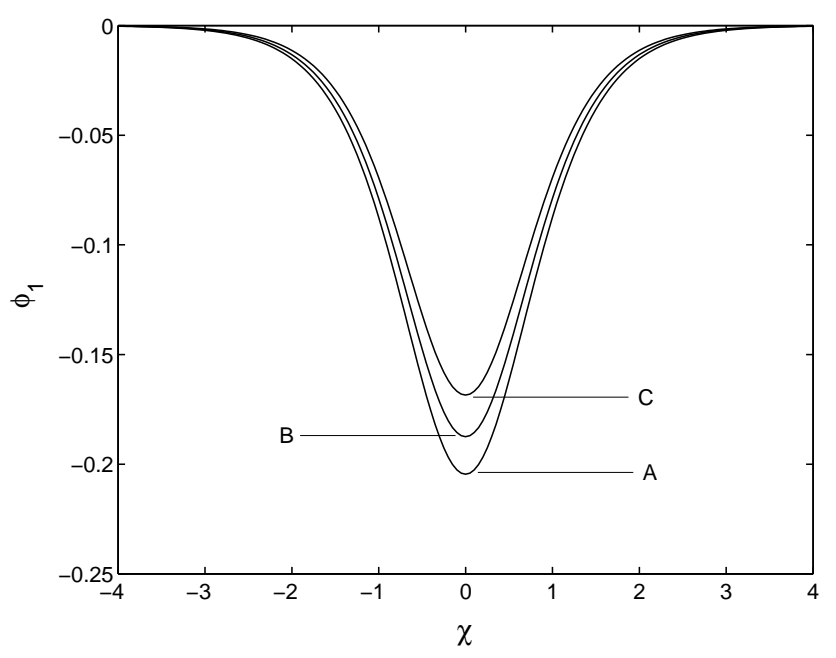

Fig. 1. Rarefactive soiltons for the parameters $\alpha=0.1, \beta=0.3$, $\mu=0.1, \theta=80^{\circ}, M=0.01$, and $\kappa=0.01$. The curves (A)-(C) correspond to $u_{0}=0.1,(V=0.175646), 0.30,(V=0.181828)$, and 0.40 , $(V=0.186529)$ respectively.

by using the boundary conditions $\phi_{1} \rightarrow 0,\left(d \phi_{1} / d \chi\right) \rightarrow 0$ and $\left(d^{2} \phi_{1} / d \chi^{2}\right) \rightarrow 0$ as $|\chi| \rightarrow \infty$, we finally obtain the following solution for the electron-acoustic soliton

$\phi_{1}=\phi_{0} \operatorname{sech}^{2}\left(\frac{\chi}{\Lambda}\right)$,

where $\phi_{0}$ and $\Lambda$ are the amplitude and width of the electron - acoustic soliton respectively, and are given by

$\phi_{0}=\frac{3 M}{A}, \quad \Lambda=\sqrt{\frac{2 B}{M}}$

For $A \neq 0$, the soliton solution given by Eq. (14) is of rarefactive type only if $A<0$. For $A>0$ solitons are of compressive nature. We have undertaken the parametric study of rarefactive solitons for different values of $u_{0}, \beta, \alpha$, and $\mu$. Equation (10) which represents the phase velocity for both rarefactive and compressive solitons has four roots. We solve Eq. (10) numerically and find that for the chosen parameters, only one positive real root exists. The other three roots are a negative real and two complex conjugate roots. Therefore, we consider only the positive real root for the phase velocity, $V$ to calculate the soliton amplitudes and widths.

In Fig. 1 we show the variation of the potential, $\phi_{1}$ with $\chi$ for different values of normalized electron beam velocity $u_{0}$. The other chosen parameters are, cold to hot ion temperature ratio, $\beta=0.3$, normalized electron beam density, $\alpha=0.1$, normalized cold ion density, $\mu=0.1, \kappa=0.01, \theta=80^{\circ}$ and $M=0.01$. The maximum amplitude of the rarefactive soliton decreases with increasing $u_{0}$. However, the width of the solitons increases with the increase in $u_{0}$.

Figure 2 shows the variation of potential, $\phi_{1}$ with $\chi$ for various values of $\beta$ and $u_{0}=0.3$. The other fixed parameters are the same as for Fig. 1. Here, we observe that as $\beta$ increases, the maximum amplitude of the soliton decreases.

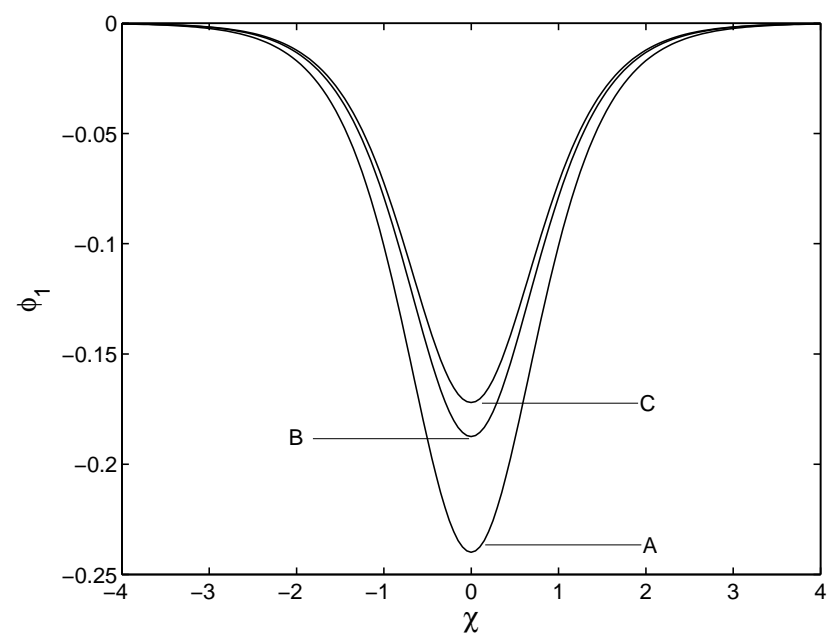

Fig. 2. Same as Fig. 1 for $\alpha=0.1, u_{0}=0.3, \quad V=0.181828$, $\mu=0.1, \theta=80^{\circ}, M=0.01$, and $\kappa=0.01$. The curves (A)-(C) are for $\beta=0.2,0.3$, and 0.4 respectively.

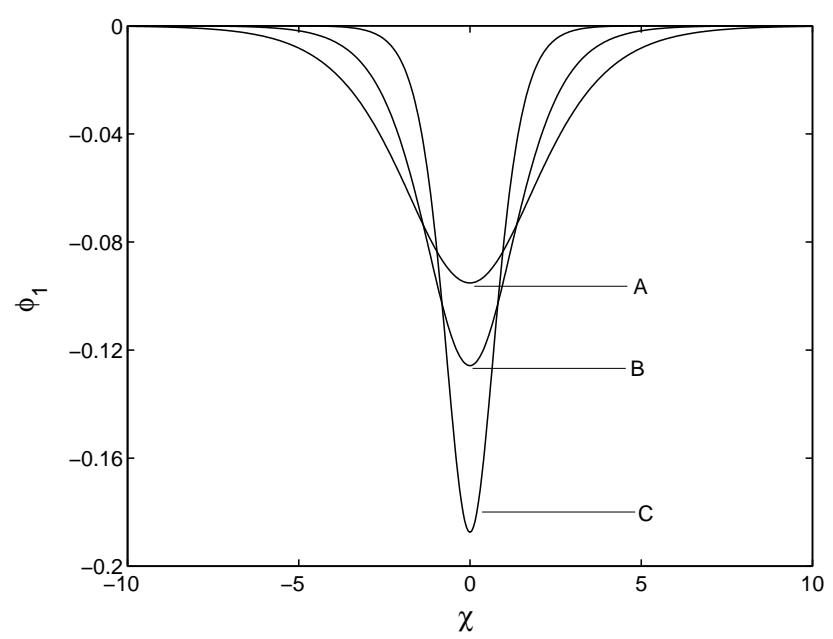

Fig. 3. Same as Fig. 1 for $\alpha=0.1, u_{0}=0.3, \mu=0.1, \beta=0.30$, $M=0.01$, and $\kappa=0.01$. The curves (A) $-(\mathrm{C})$ are for , $\theta=70^{\circ}$ $(V=0.358132), 75^{\circ}(V=0.271011)$, and $80^{\circ}(V=0.181828)$ respectively.

On the other hand, width of the solitons decreases with the increase in $\beta=T_{l} / T_{h}$.

Figure 3 shows the variation of the soliton amplitude with $\chi$ for various values of angle $\theta$ between the propagation direction and the ambient magnetic field $\boldsymbol{B}_{\mathbf{0}}$, as shown on the curves. It is seen that as the angle increases the amplitude of the soliton increases and the width of the soliton decreases.

Since, we have neglected the thermal effects of electrons, cold electron temperature does not appear explicitly in our model. To satisfy the assumption of cold electrons, one must have $T_{e}<<T_{e f}$, where $T_{e}$ is the electron temperature. The velocity of the rarefactive solitons is given by $V_{r s}=V+M \epsilon$, where $\epsilon$ is a smallness parameter. 
For the typical chosen parameters of the plasma sheet boundary layer, namely, the hot - ion temperature, $T_{h}=1 \mathrm{keV}$, the cold - ion temperature, $T_{l}=100 \mathrm{eV}$, $B_{0}=25 \mathrm{nT}$, which correspond to the near-Earth magnetotail (Lui et al., 1991) $\alpha=\mu=0.1, \beta=0.1, \theta=80^{\circ}, M=0.004$, $\epsilon=0.1$ and electron beam velocity $u_{0} \sim 3800 \mathrm{~km} / \mathrm{s}$, the effective ion temperature is found to be $T_{e f} \sim 525 \mathrm{eV}$, the associated soliton velocity is found to be of the order of $V_{r s} \sim 1800 \mathrm{~km} / \mathrm{s}$. The corresponding normalized electric field, $E=-d \phi_{1} / d \chi$, of the soliton structures can be obtained by differentiating Eq. (14) with respect to $\chi$. Then, for the above mentioned parameters, the absolute value of the electric field is estimated to be in the range $\sim(3-40) \mathrm{mV} / \mathrm{m}$. The width, $\Lambda$, of the rarefactive solitons is given by Eq. (15) and is found to be $\sim 3.4 \mathrm{~km}$ for the above mentioned parameters.

\section{Conclusions}

We have studied the nonlinear propagation of electronacoustic waves in a magnetized plasma consisting of cold electron beam and background plasma electrons and two types of ions, i.e. the cold and hot ions with Boltzmann distributions.

It is emphasized that amplitude of the electron-acoustic solitons as well as parametric regime where the solitons can exist is sensitive to the electron beam velocity, electron beam density, cold to hot ion temperature ratio, $\beta$ and the cold ion density. The electron-acoustic soliton amplitude for rarefactive solitons decreases with the increase in the cold to hot ion temperature ratio.

The model presented here may be applied to explain some feature of the low-frequency component of BEN observed in the plasma sheet boundary layer. It is found that the lowfrequency waves are polarized nearly perpendicular to the background magnetic field (Gurnett et al., 1976; Onsager et al., 1993). This feature of the low-frequency broadband electrostatic waves could be explained naturally by our model as the electron-acoustic solitons propagate at large angle to $\boldsymbol{B}_{\mathbf{0}}$. However, the theory cannot apply to the high-frequency part of BEN (Onsager et al., 1993) or to solitary structures observed by Geotail (Matsumoto et al., 1994) as their electric fields are polarized parallel to $\boldsymbol{B}_{\mathbf{0}}$, and also they have much higher frequencies than the electron-acoustic modes studied here.

In the present model, the electrons are treated as cold. This assumption demands electron temperature $T_{e}$ to be much smaller than effective ion temperature $T_{e f}$, i.e. $T_{e}<<T_{e f}$. The model can be generalized by including the electron thermal effects.
Acknowledgements. GSL would like to thank Y. Kamide for the kind hospitality provided during his stay at Solar-Terrestrial Environment Laboratory, Nagoya University, Japan. SVS is thankful to H. Matsumoto for the hospitality at RASC, Kyoto University, Japan and JSPS for financial support.

Edited by: J. Büchner

Reviewed by: one referee

\section{References}

Buti, B.: Nonlinear electron-acoustic waves in a multi-species plasma, J. Plasma Phys., 24, 169-180, 1980.

Buti, B., Mohan, M., and Shukla, P. K.: Exact electron-acoustic solitarywaves, J. Plasma Phys., 24, 341-347, 1980.

Cattell, C., Dombeck, J., Wygant, J. R., Hudson, M. K., Mozer, F. S., et al.: Comparisions of polar satellite observations of solitary wave velocities in the plasma sheet boundary and the high altitude cusp to those in the auroral zone, Geophys. Res. Lett., 26, 425-428, 1999.

Ergun, R. E., Calrson, C. W., McFadden, J. P., Mozer, F. S., Delory, G. T., et al.: FAST satellite observations of large-amplitude solitary structures, Geophys. Res. Lett., 25, 2041-2044, 1998.

Guha, S. and Dwivedi, C. B.: Linear and nonlinear propagation ofelectron-acoustic waves in a multi-species plasma, J. Plasma Phys., 32, 283-290, 1984.

Gurnett, D. A., Frank, L. A., and Lepping, R. P.: Plasma waves in the distant magnetotail, J. Geophys. Res., 81, 6059-6071, 1976.

Kojima, H., Matsumoto, H., Miyatke, T., Nagano, I., Fujita, A., et al.: Relation between electrostatic solitary waves and hot plasma flow in the plasma sheet boundary layer: Geotail observations, Geophys. Res. Lett., 21, 2919-2922, 1994.

Lui, A. T. Y., Chang, C. L., Mankofsky, A., Wong, H. K., and Winske, D.: A cross-field current instability for substorm expansions, J. Geophys. Res., 96, 11 389-11 401, 1991.

Mace, R. L., Baboolal, S., Bharuthram, R., and Hellberg, M. A.: Arbitrary amplitude electron-acoustic solitons in a two electroncomponentplasma, J. Plasma Phys., 45, 323-338, 1991.

Matsumoto, H., Kojima, H., Miyatake, T., Omura, Y., Okada, M., et al.: Electrostatic solitary waves (ESW) in the magnetotail: BEN wave forms observed by GEOTAIL, Geophys. Res. Lett., 21, 2915-2918, 1994.

Onsager, T. G., Thomsen, M. F., Elphic, R. C., Gosling, J. T., Anderson, R. R., et al.: Electron generartion of electrostatic waves in the plasma sheet boundary layer, J. Geophys. Res., 98, 15 509$15519,1993$.

Parks, G. K., McCarthy, M., Fitzenreiter, R. J., Etcheto, J., Anderson, K. A., et al.: Particle and field characteristics of the high latitude plasma sheet boundary layer, J. Geophys. Res., 89, 88858906, 1984.

Reddy, R. V. and Lakhina, G. S.: Ion acoustic double layers and solitons in auroral plasma, Planet. Space Sci., 39, 1343-1350, 1991.

Washimi, H. and Taniuti, T.: Propagation of ion-acoustic solitarywaves of small amplitude, Phys. Rev. Lett., 17, 996-998, 1966.

Yu, M. Y. and Shukla, P. K.: Linear and nonlinear electron-acoustic waves, J. Plasma Phys., 29, 409-413, 1983. 